\title{
DAY CASE MANAGEMENT IN ADJUSTABLE SUTURE SQUINT SURGERY
}

\author{
A. J. LUFF' ${ }^{1}$, R. J. MORRIS ${ }^{1}$ and A. C. WAINWRIGHT ${ }^{2}$ \\ Southampton
}

\begin{abstract}
SUMMARY
Day case adjustable suture squint surgery is limited by patient cooperation in the early post-operative period. Nausea is common and may be exacerbated by adjustment. To facilitate early adjustment, in $\mathbf{3 7}$ consecutive patients anaesthesia was induced with propofol and the airway maintained with a largyngeal mask. Before and after adjustment patients recorded their level of nausea on a visual analogue scale $(1=$ no nausea, $10=$ vomiting). The mean age of the group was 31.9 years with 20 men and 17 women. Adjustment was performed at a mean time of 4.9 hours after surgery. On the analogue scale of nausea the mean score was 1.54 pre-adjustment and 1.73 post-adjustment. Only 1 patient was given a post-operative anti-emetic. All patients were sufficiently alert to adjust without difficulty and were discharged the same day. The use of a laryngeal mask and induction of anaesthesia with propofol in adjustable suture squint surgery facilitates early adjustment and thus day case management.
\end{abstract}

Increasing awareness of the advantages of day case management is pervading all branches of surgery. Ophthalmologists have adopted this practice for many common surgical procedures, most of which are performed under local anaesthesia. Squint surgery, however, usually involves general anaesthesia and although a general anaesthetic per se does not contraindicate day case management, ${ }^{1}$ difficulties have been encountered. A 1977 report on day case surgery for strabismus in children concluded that vomiting and drowsiness were the main postoperative problems. ${ }^{2}$ The situation had changed little by the beginning of this decade when a study on strabismus patients of all ages showed that $7.9 \%$ of intended day cases required overnight admission and that the most common cause (38\% of cases) was nausea. ${ }^{3}$ The relevance of the side effects of general anaesthesia is magnified when adjustable suture squint surgery is contemplated, as

From: 'The Eye Hospital, Southampton; ${ }^{2}$ Department of Anaesthesia, Southampton General Hospital, Southampton, UK.

Correspondence to: Mr. R. J. Morris, The Eye Hospital, Wilton Avenue, Southampton SO9 4XW, UK. patient compliance and cooperation are paramount. Vagal effects of extraocular muscle manipulation ${ }^{4}$ are well known to ophthalmologists, and serve to compound the difficulties faced in early post-operative suture adjustment. ${ }^{5}$

In an attempt to overcome these difficulties we have carried out adjustable suture squint surgery using anaesthetic methods known to promote swift recovery with minimal discomfort. Propofol is an intravenous anaesthetic agent used to induce (and maintain) anaesthesia, from which recovery is rapid and nausea minimal. ${ }^{6}$ The laryngeal mask is an increasingly popular device designed to reduce the complications associated with conventional endotracheal intubation. ${ }^{\text {? }}$

\section{PATIENTS AND METHODS}

A prospective observational study was carried out on 37 consecutive patients undergoing adjustable suture squint surgery. All were planned as day cases, surgery being carried out during the morning with adjustment performed in the afternoon. Premedication was avoided in order to increase post-operative awareness and decrease any potential nausea. Anaesthesia was induced with propofol and maintained with enflurane and nitrous oxide. A laryngeal mask was employed to maintain the airway. No routine intra-operative anti-emetic was used and post-operative analgesia and anti-emesis were administered 'as required' by the nursing staff who were unaware of the trial. Patients were encouraged to eat and drink as they felt fit prior to adjustment.

Adjustment was performed in a side room which necessitated patients walking a distance of at least 20 metres. Before adjustment a record of post-operative medication was made, and the patient asked to score the degree of nausea experienced. This was done on a visual analogue scale with a range of 1 to 10: a score of 1 indicated that the patient was completely well and a score of 10 that the patient was vomiting. A second 'nausea score' was taken on completion of adjustment.

At the end of the afternoon patients were assessed, with a view to discharge should they be sufficiently alert and 
comfortable. All patients were asked to comment on any specific non-ocular discomfort they had experienced.

\section{RESULTS}

Of the 37 patients studied 20 were men and 17 women. The mean age was 31.9 years (range 8-71 years). In 10 cases binocular surgery was performed: in 8 of these patients a single muscle was adjustable and in 2 of them two muscles were adjustable. In 27 cases uniocular surgery was performed: in 25 of these patients a single muscle was adjustable and in 2 of them two muscles were adjustable. The mean number of muscles operated on was 1.8 per patient. The mean duration of anaesthesia was 53 minutes (SD 18 minutes, range 25-90 minutes).

Twenty of the patients received post-operative analgesia. In 17 cases this amounted to a single oral dose of paracetamol; 2 patients received intramuscular Omnopon (Roche) and 1 patient intramuscular pethidine. Only one patient was given an anti-emetic (cyclizine) and this was as a routine prophylactic accompaniment of intramuscular pethidine. The mean time to adjustment was 4.9 hours (SD 1.3 hours, range $2-7$ hours).

The mean pre-adjustment nausea score on the analogue scale was 1.54 (SD 0.99, range 1-5). This increased to a mean post-adjustment nausea score of 1.73 (SD 1.73, range 1-10). Twenty-nine patients maintained the same pre- and post-adjustment scores, in 23 cases this score being 1 ('completely well') on the visual analogue scale.

During adjustment the nausea score 'deteriorated' in 4 cases (mean increase of 3.5 on the analogue scale, range $1-9$ ) and 'improved' in 4 cases (mean decrease of 1.5 on the analogue scale, range 1-2). Vomiting was induced in 1 patient.

None of the patients was aware of any adverse reaction to anaesthesia, all claimed to feel clear-headed, all cooperated well with assessment and adjustment (including a cover test and examination of ocular versions) and all were considered fit for discharge the same day.

\section{DISCUSSION}

Strabismus surgery causes vomiting more frequently than any other type of ocular surgery. ${ }^{8}$ As the addition of powerful anti-emetics to conventional anaesthesia proved unconvincing ${ }^{8,9}$ we have focused our attention on the use of alternative anaesthetic regimens. A study on rapidity of recovery and post-operative vomiting following squint surgery in children showed total intravenous anaesthesia (induction and maintenance) with propofol to be superior to halothane. ${ }^{10}$ It seems logical, therefore, to assess the use of propofol in adjustable suture squint surgery where any tendency to nausea appears to be exacerbated by extraocular muscle manipulation ${ }^{5}$ and patient compliance is vital.

Propofol (2,6-diisopropylphenol) is an intravenous agent used for the induction and maintenance of general anaesthesia. Anaesthesia is induced with few excitatory effects, ${ }^{11}$ recovery is rapid and clear-headed,${ }^{12}$ there is a short interval between awakening and orientation ${ }^{6}$ and a low incidence of post-operative nauséa and vomiting. ${ }^{6,13,14}$ Propofol is more expensive than traditional inhalational anaesthetic agents but there is evidence that using propofol simply as an induction agent (and maintaining anaesthesia with enflurane) results in rapid recovery and a low incidence of nausea and vomiting. ${ }^{12,15}$ This method was therefore selected for use in our study.

No specific problems have been encountered with propofol in ophthalmic usage. ${ }^{16}$ Nasal hypersecretion sufficient to contaminate the surgical field has been reported in 2 patients in whom anaesthesia was maintained with propofol, ${ }^{17}$ but was not noted in any of our patients. Cardiorespiratory depression, a known adverse reaction to propofol, ${ }^{11}$ did not occur. The laryngeal mask $^{7}$ has been shown to offer a safe alternative to conventional intubation with the potential of greater comfort in the postoperative period.

Exponents of adjustable suture squint surgery have described adjustment on the morning following surgery, ${ }^{18}$ citing post-operative vagal responses as a contraindication to early adjustment. ${ }^{19}$ The aim of our study was to observe the feasibility of day case adjustable surgery by employing anaesthetic measures specifically designed to facilitate early adjustment. Investigation of the recovery of muscle tone after propofol suggests that ocular alignment should stabilise in the early post-operative period: electromyelographic studies have shown no additive effect of propofol on recovery from neuromuscular blockade,$^{20}$ and muscle balance testing with the Maddox Wing demonstrated a return to baseline values within 3 hours of total intravenous anaesthesia with propofol ${ }^{13}$ The possibility of day case management by operating under local anaesthesia with on-table adjustment has been explored ${ }^{21}$ but the indications are acknowledged to be limited. ${ }^{22}$

Inducing anaesthesia with propofol and using a laryngeal mask as routine practice we were able to perform day case adjustable suture squint surgery on 37 consecutive cases. No routine anti-emetic was given (1 patient received cyclizine prophylactically with intramuscular opiate) and mean visual analogue 'nausea scores' were less than 2 (on a scale of 1 to 10) both before and after adjustment. One patient, an 11-year-old girl, vomited having felt completely well prior to adjustment. Nausea was induced in this case when the adjustable muscle was recessed; the globe was anchored and the patient instructed to look in the direction of action of the relevant muscle. It may be that this particular manoeuvre, if anticipated, should be preceded by routine administration of an anti-emetic regardless of the degree of pre-adjustment nausea.

The apparent awareness and lack of nausea in these 37 cases will, for the moment, make the use of propofol and a laryngeal mask routine for adjustable suture squint surgery in our unit. The principal objection to the use of propofol is expense, but this is minimal and must be balanced against decreased patient discomfort and the inconvenience and financial implications of a potentially unnecessary overnight hospital stay. 
Key words: Adjustable sutures, Day case surgery, Laryngeal mask, Propofol.

\section{REFERENCES}

1. Commission on the Provision of Surgical Services. Guidelines for day case surgery. London: Royal College of Surgeons of England, 1985.

2. Hadaway EG, Ingram RM, Traynar MJ. Day case surgery for strabismus in children. Trans Ophthalmol Soc UK 1977; 97:23-5.

3. Isenberg SJ, Apt L, Yamada S. Overnight admission of outpatient strabismus patients. Ophthalmic Surg 1990;21: 540-3.

4. Apt L, Isenberg S, Gaffney WL. The oculocardiac reflex in strabismus surgery. Ám J Ophthalmol 1973;76:533-6.

5. Eustis HS, Eiswirth CC, Smith DR. Vagal responses to adjustable sutures in strabismus correction. Am J Ophthalmol 1992;114:307-10.

6. Sanderson JH, Blades JF. Forum: Multicentre study of propofol in day case surgery. Anaesthesia 1988;43 (Suppl): 70-3.

7. Brain AIJ, McGhee TD, McAteer EJ, Thomas A, Abu-Saad MAW, Bushman JA. The laryngeal mask airway. Anaesthesia 1985;40:356-61.

8. Van Den Berg AA, Lambourne A, Yazji NS, Laghari NA. Vomiting after ophthalmic surgery. Anaesthesia 1987;42: 270-6.

9. Van Den Berg AA, Lambourne A, Clyburn PA. The oculoemetic reflex. Anaesthesia 1989;44:1 10-7.

10. Watcha MF, Simeon RM, White PF, Stevens JL. Effect of propofol on the incidence of postoperative vomiting after strabismus surgery in pediatric outpatients. Anesthesiology 1991;75:204-9.

11. MacKenzie N, Grant I. Comparison of the new emulsion formulation of propofol with methohexitone and thiopentone for induction of anaesthesia in day cases. $\mathrm{Br} \mathrm{J}$ Anaesth 1985;57:725-31.
12. Grant IS, MacKenzie N. Recovery following propofol ('Diprivan') anaesthesia: a review of three different anaesthetic techniques. Postgrad Med J 1985;61 (Suppl 3):133-7.

13. Kay B, Healy TEJ. Propofol ('Diprivan') for outpatient cystoscopy: efficacy and recovery compared with althesin and methohexitone. Postgrad Med J 1985;61 (Suppl 3):108-14.

14. Gunawardene RD, White DC. Propofol and emesis. Anaesthesia 1988;43 (Suppl):65-7.

15. Mirakhur RK, Elliott P, Stanley JC. Use of propofol in anaesthesia for ophthalmic surgery. In: Prys-Roberts C, editor. Focus on infusion: intravenous anaesthesia. London: Current Medical Literature Ltd, 1991:134-9.

16. Mirakhur RK, Shepherd WFI. Intraocular pressure changes with propofol ('Diprivan'): comparison with thiopentone. Postgrad Med J 1985;61 (Suppl 3):41-4.

17. Bautista DV, Keech RV. Propofol and surgical contamination in strabismus surgery. Am J Ophthalmol 1992;114: 635.

18. Kushner BJ. Pearls and pointers in adjustable sutures for strabismus surgery. Transactions of symposium on oculoplastic surgery, strabismus and pediatric ophthalmology. New Orleans Academy of Ophthalmology. Thorofare, NJ: Slack, 1990:215-25.

19. Kushner BJ. Vagal responses to adjustable sutures in strabismus correction [correspondence]. Am J Ophthalmol 1993;115:124-5.

20. Nightingale P, Petts NV, Healy TEJ, Kay B, McGuiness K. Induction of anaesthesia with propofol ('Diprivan') or thiopentone and interactions with suxamethonium, atracurium and vecuronium. Postgrad Med J 1985;61 (Suppl 3):31-4.

21. Thorson JC, Jampolsky AJ, Scott AB. Topical anaesthesia for strabismus surgery. Trans Am Acad Ophthalmol Otolaryngol 1966;70:968-72.

22. Ruben ST, Elston JS. One stage adjustable sutures: practical aspects. Br J Ophthalmol 1992;76:675-7. 